\title{
Application of Vacuum Photoelectric Detection Technology in Super-Resolution System
}

\author{
Kai Gu, Xuefeng Liu*, Yang Zhang, Hanwen Zhao, Weiping Liu \\ School of Electronic and Optical Engineering, Nanjing University of Science and Technology, Nanjing, China \\ Email: *liuxf1956@163.com
}

How to cite this paper: Gu, K., Liu, X.F. Zhang, Y., Zhao, H.W. and Liu, W.P. (2020) Application of Vacuum Photoelectric Detection Technology in Super-Resolution System. Optics and Photonics Journal, 10, 141-148.

https://doi.org/10.4236/opj.2020.106015

Received: June 5, 2020

Accepted: June 26, 2020

Published: June 29, 2020

\begin{abstract}
Due to the wave characteristics of light, diffraction occurs when the light passes through the optical system, so that the resolution of the ordinary far-field optical system is limited by the size of the Airy disk diameter. There are various factors that cause image quality degradation during system detection and imaging, such as optical system aberrations, atmospheric interference, defocusing, system noise and so on. Super-resolution optical imaging technology is the most innovative breakthrough in the optical imaging and detection field in this century. It goes beyond the resolution limit of ordinary optical systems or detectors, and can get more details and information of the structure, providing unprecedented tools for various fields. Compared with ordinary optical systems, super-resolution systems have very high requirements on the signals to be detected, which cannot be met by ordinary detection techniques. Vacuum photoelectric detection and imaging technology is equipped with the characteristics of high sensitivity and fast response. It is widely used in super-resolution systems and has played a great role in super-resolution systems. In this paper, the principles and structure of the image-converter streak camera super-resolution system, scanning electron microscopy super-resolution system and laser scanning confocal super-resolution system will be sorted out separately, and the essential role of the vacuum photoelectric detection technology in the ultra-microscopic system will be analyzed.
\end{abstract}

\section{Keywords}

Diffraction, Image Quality, Super-Resolution System, Vacuum Photoelectric Detection

\section{Introduction}

With the development of bioscience, high energy physics and other fields, people 
have higher and higher requirements for spatial and temporal resolution. Therefore, more and more emphasis has been placed on super-resolution technology for the last few years. Super-resolution technology, being a cutting-edge research topic in the field of optical engineering, not only involves in the design of optical imaging system, but also concerns in the field of image acquisition and processing. According to the signal generation and transmission process, the resolution of the system can be regarded as consisting of three parts [1], including diffraction resolution, geometric resolution, and noise equivalent resolution. People improve the above three resolutions by improving the optical system, improving the image processing ability, etc., in order to achieve the purpose of super resolution. Photoelectric detection is an indispensable link between a super-resolution system. Therefore, optimizing and improving the photoelectric detection capability helps to achieve the final imaging result of the system. In view of the high requirement of the super-resolution system for detected signals, ordinary detection technology cannot meet its requirements. Nevertheless, vacuum photoelectric detection technology is extensively applied to the super-resolution system on account of its high sensitivity and Short relaxation process [2], and played significant effect of the super-resolution system. This article lists three super-resolution systems and discusses the application of vacuum photoelectric detection and imaging technology.

\section{Image Converted Tube Streak Camera Super-Resolution System}

\subsection{Basic Working Principle of the System}

The working principle of the image converted tube streak camera is shown in Figure 1. As shown in the figure, the deflection system and micro channel plate are placed in the image converted tube. The objective lens system in the system can image the transient light source in a narrow slit, so the one-dimensional information of the image can be obtained. The imaging lens system images this one-dimensional information on the photocathode, from where the photoelectrons
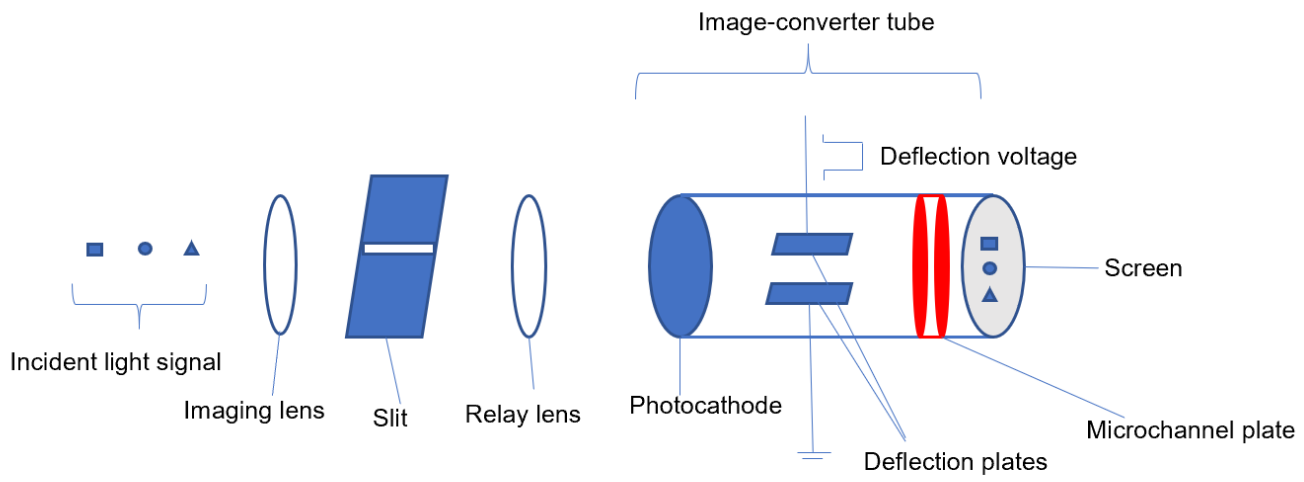

Figure 1. Working principle of the image-converter streak camera. 
are emitted, passing through the focusing system and entering the deflection system with slope voltage which changes linearly from time. As a result, the electrons that enter the deflection system chronologically are subjected to different deflection voltages. Electron multiplication can be realized when the electrons incident at different time pass through the deflection system to the micro channel plate. The multiplicative electron bombards the fluorescent screen that converts the electronic image of visible light. Because of the different incident time, the time for the multiplied electrons to reach the screen is different. These electrons will be distributed among the direction perpendicular to the slit, so the position information on the screen is transformed into the time information. In other words, the different positions of electrons incident on the screen represent different incident times [3] [4].

As a high time-resolution device, image converted tube streak camera has important applications for various scientific research fields. Time-resolution, which is defined as the minimum time interval between the detected light pulses, is its most important performance parameter. Because of the limitation of the resolution of the imaging system, the final image formed by the electrons on the screen is diffuse, which not only leads to a reduction in the resolution of the imaging system, but also causes a decrease in the temporal resolution to the time domain. The super-resolution restoration technology can be applied to the imaging results of the image converted tube streak camera, processing the image obtained by the imaging system and obtaining the super-resolution image. This technology can transform the spatial super resolution of temporal resolution, and further improve the camera's temporal resolution.

The ordinary imaging system can be regarded as a low-pass filter. When it is greater than the cut-off frequency, the transfer function is zero, and high-frequency information is limited. Therefore, in order to achieve super-resolution, we must get the information above the cut-off frequency [5] [6] [7]. Using a super-resolution restoration technique, information above the cut-off frequency can be obtained. According to the information superposition theory, the information above the cut-off frequency is superposed into the frequency component below the cut-off frequency by convolution. In other words, the frequency component below the cut-off frequency contains all the information about the object of a bounded restricted object. That is, for a bounded restricted object, the frequency component below the cutoff frequency contains all the information on the object. Then, the analytic continuation principle can be used to reconstruct the information above the cut-off frequency using signals below the cut-off frequency, so as to realize the super-resolution of the image and improve the temporal resolution of the camera.

\subsection{Application of Vacuum Photoelectric Technology in the System}

The Image converted tube streak camera super-resolution system is an optical 
system, utilizing the super-resolution recovery technology to make its time super-resolution higher, composed of the image converter tube, micro channel plate and so on, and combined with other components and technologies.

The image converter is a kind of vacuum imaging device which can transform the invisible light image into the visible light band, including three parts: photocathode, electronic acceleration system and electro-optic conversion fluorescent screen. When the invisible ray such as X-ray is incident on the photocathode, photoelectrons will be excited. The photoelectrons are accelerated by the electron acceleration system, which is imaged on the fluorescent screen to enhance the brightness of the image, so that the fluorescent screen emits much stronger light energy. Microchannel plate is also a vacuum electron multiplier device, which has the function of compensating and enhancing electrons and has high time resolution. A semiconductor material that can emit secondary electrons is coated from the inner wall of the micro channel plate, and a direct voltage is applied across the micro channel plate to form a uniform electric field. When low-energy photoelectrons touch the inner wall of the micro channel plate, secondary electrons are excited. The excited secondary electrons are accelerated under the action of a uniform electric field, and then further touch the inner wall to achieve electron multiplication and the effect of enhancing the photoelectron image.

\section{Scanning Electron Microscopy Super-Resolution System}

\subsection{Basic Working Principle of the System}

Scanning electron microscope can be divided into six systems, including vacuum system, electronic optical system, signal detection amplification and display system, image recording system, cooling circulating water system and power supply system [8] [9]. The working principle is as follows: The electron gun in the electron optical system emits a high-brightness and small beam diameter electron beam, which then is focused on the sample surface in the sample chamber by the electromagnetic lens in the electron optical system. After scanning on the sample surface by the scanning coil, the high-energy incident electron beam interacts with the sample to generate different signals such as secondary electrons. Scanning electron microscopy is carried out using the image decomposition method of point-by-point imaging, that is, the signal emitted from any point in the surface of the sample corresponds to the corresponding bright spot on the fluorescent screen of the kinescope. Because the scanning electron microscope uses the electron beam as the medium, it has broken through the limitation of the diffraction limit of ordinary microscopes when visible light is used as the medium. The scanning electron microscope can magnify the sample of more than 10,000 times, achieving extremely ultra-high resolution.

\subsection{Application of Vacuum Photoelectric Technology in the System}

The three main systems in scanning electron microscope are electronic optical 
system, signal detection amplification and display system and vacuum system. The electronic optical system and signal detection amplification and display system, whose working environment requires a high vacuum environment, are all placed in the vacuum system. Therefore, these three systems can be considered as a large vacuum photoelectric detector. The first reason why it is necessary to change the system into a vacuum is to protect the filament in the electron gun of the electronic optical system. Once the filament is exposed to the air, it will be rapidly oxidized and fail to emit electrons. Moreover, the vacuum degree of the system has a great relationship of the electron production efficiency of the electron gun. Secondly, it is conducive to increase the average free path of the electron, so as to obtain more electrons for imaging.

In the signal detection amplification and display system, owing to the interaction of the electron beam and the sample, different types of signals, such as secondary electrons, Auger electrons, and X-rays, are generated. Consequently, different detectors are required to receive the signals. One of the most commonly used in scanning electron microscopes is the electron detector, which is composed of scintillator, light pipe and photomultiplier. The photomultiplier tube is also a small vacuum photodetector. A typical photomultiplier tube is in a vacuum tube, containing the entrance window, photocathode, focusing electrode, electron multiplier stage and electron collector. The photon is transmitted to the photomultiplier by the optical guide tube for amplification and converted into a current signal output, which is amplified by the video amplifier and becomes a modulation signal.

\section{Laser Scanning Confocal Super-Resolution System}

\subsection{Basic Working Principle of the System}

The working principle of laser scanning confocal microscope is shown in Figure 2. The laser scanning confocal microscope takes the laser as the light source. Because of the excellent monochromatism and directivity of the laser, the chromatic aberration appears in the system imaging is eliminated fundamentally. In addition, the system adopts confocal technology, the specific principle of which is as follows: the detector and the excitation light source are placed in different light paths by spectroscope, and a baffle is placed in front of the light source. The baffle is placed at the focal plane of the objective lens, and a small hole is punched in the center of which to form an illuminated pinhole. After the laser passing through the illuminated pinhole, it can form a point light source with strong directivity, small divergence and high brightness, thus eliminating the spherical aberration after focusing. A focused laser beam is used to scan the sample point by point. If there are substances in the sample that can be excited by the laser, the substance in the sample is excited and emits fluorescence which will be focused on the focal plane, where a central perforated baffle plate is placed. The baffle plate is placed in front of the detector, which ensures that only the fluorescence signal excited by the laser focusing at the sample point can pass 


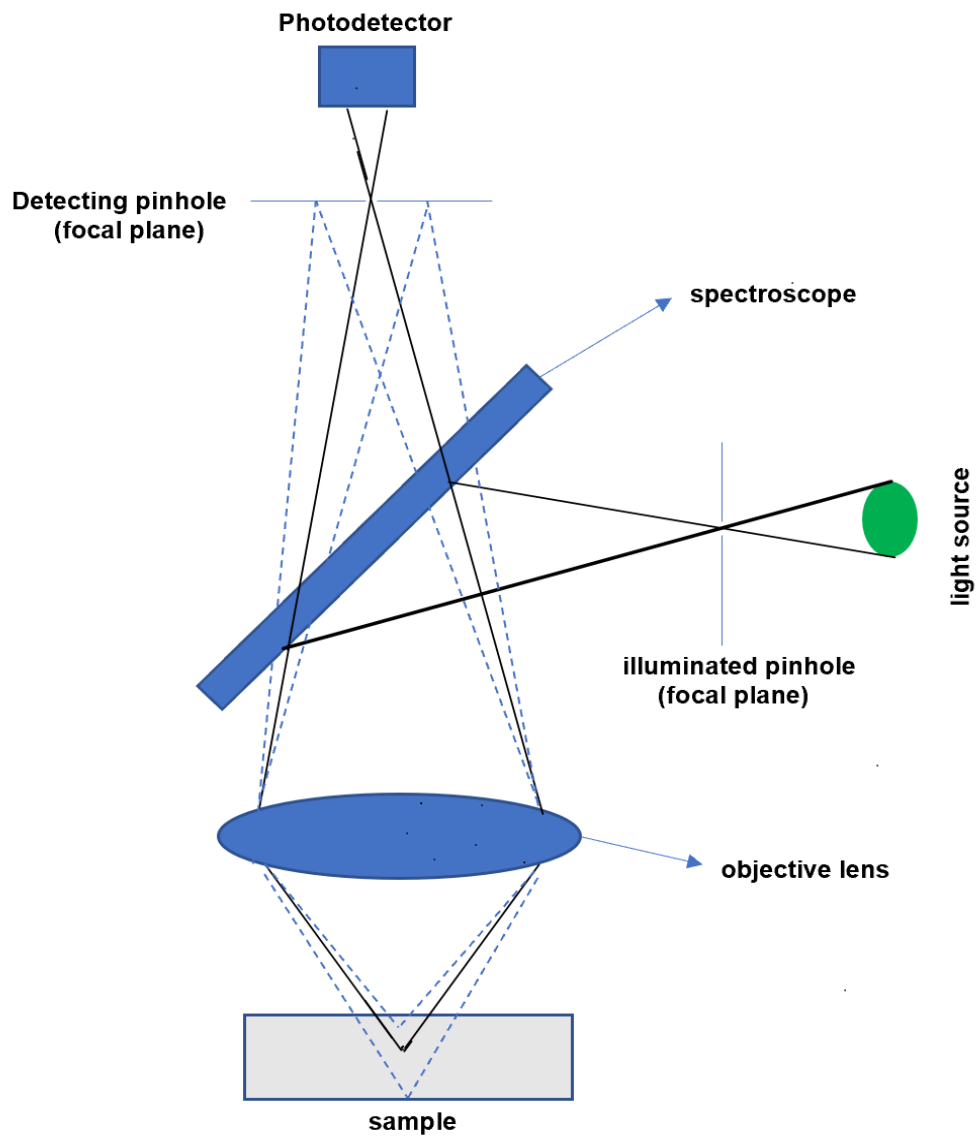

Figure 1. Working principle of laser scanning confocal microscope.

through the pinhole. Compared with the traditional optical microscope imaging under the field light source, in the laser scanning confocal super-resolution system, the detector collects only the fluorescence signal at the focal point that will not be disturbed by the diffraction or scattering of other points outside the focus point every time. Then by scanning each point on the sample in sequence, you can get the signals of countless points on the sample. These signals are converted into electrical signals and transmitted to a computer, and which then processed, analyzed and output the signals to form a complete two-dimensional or three-dimensional image. The resolution and precision of laser confocal system are greatly improved compared with ordinary microscope [10]. So as to receive electrical signals point by point, amplify weak signals and improve the sensitivity to the signal, the system generally uses photomultiplier tubes in vacuum photodetectors.

\subsection{Application of Vacuum Photoelectric Technology in the System}

In the traditional laser scanning confocal super-resolution system, a common photomultiplier tube is generally used to detect the signal. But for improving the resolution of system imaging, it is necessary to increase the power and time of 
laser irradiation, but this will reduce the survival rate of the sample. If you want to reduce the phototoxicity, you must reduce the power and time of the laser irradiation, which will prevent the ordinary photomultiplier tube from collecting the fluorescent signal. In order to solve this contradiction, a hybrid photodetector, which can better collect more fluorescent signals while using a lower power laser, can be used to take the place of the traditional photomultiplier tube.

The new hybrid photodetector incorporates a solid-state semiconductor anode chip into a traditional photomultiplier tube, which hits off the disadvantages of low signal-to-noise ratio and easy saturation of signal of the micro channel plate in the traditional photomultiplier tube, and realizes that the effect of doubling electrons is enhanced, and broadens the use of vacuum photoelectric devices. In the new laser scanning confocal super-resolution system, a gallium arsenide phosphide (GaAsP) hybrid photodetector is generally used [11].

Conventional laser confocal microscopy uses a photomultiplier detector. When photons are incident on the photocathode, photoelectrons are obtained. These electrons hit the anode through the action of high voltage to achieve electron multiplication. In the new GaAsP hybrid detector, incident photons generate photoelectrons through the gallium arsenide phosphorous photocathode. These photoelectrons bombard the semiconductor chip at a very high speed under a voltage acceleration much higher than the acceleration voltage in the photomultiplier tube. The anode, which ultimately produces electron bombardment gain and avalanche gain, obtains better excitation effects than ordinary photomultiplier tubes [12]. Due to its higher sensitivity and lower background noise, a lower intensity laser can be used to illuminate the sample, which not only ensures the survival rate of cells, but also ensures the collection of fluorescent signals.

\section{Conclusion}

Vacuum photoelectric imaging and detection technology, due to its high sensitivity and fast response, has been widely used in super-resolution systems, and has played a great role in super-resolution systems. This article briefly summarizes the role of vacuum photoelectric detection technology in three super-resolution systems, and analyzes the role of related devices and technologies in different super-resolution systems. Finally, it is concluded that vacuum photoelectric technology is essential in the super-resolution system.

\section{Acknowledgements}

Upon the completion of this thesis, first of all, I would like to express my heartfelt thanks and respect to my mentor, Prof. X. Liu. I would also like to express my heartfelt thanks to all the people who care for and help me. My senior Yang Zhang, Hanwen Zhao and Weiping Liu gave me valuable advice during my writing and provided me with answers to relevant professional questions. At the same time, I would like to thank my entire research team for their support for my participation in this meeting. 


\section{Conflicts of Interest}

The authors declare no conflicts of interest regarding the publication of this paper.

\section{References}

[1] Zalevsky, Z. and Mendlovic, D. (2004) Optical Superresolution. Springer, New York. https://doi.org/10.1007/978-0-387-34715-8

[2] Chen, J.Z. (1994) The Development of Vaccum Photoelectronic Detection Devices and Their Application. Journal of Applied Optics.

[3] Niu, L.H., Yang, Q.L., Niu, H.B., Liao, H. and Zhou, J.L. (2008) A Wide Dynamic Range X-Ray Streak Camera System. Review of Scientific Instruments, 79, 023103. https://doi.org/10.1063/1.2839025

[4] Shakya, M.M. and Chang, Z. (2004) An Accumulative X-Ray Streak Camera with 280-fs Resolution. Proceedings of SPIE International Society for Optical Engineering, 5534, 125-131. https://doi.org/10.1117/12.560526

[5] Tescher, A.G. (1991) Imagery Super-Resolution: Emerging Prospects. Proceedings of SPIE International Society for Optical Engineering, 1567.

[6] Hunt, B.R. (1999) Super-Resolution of Imagery: Understanding the Basis for Recovery of Spatial Frequencies beyond the Diffraction Limit. Information, Decision and Control, IDC 99, Proceedings. https://doi.org/10.1109/IDC.1999.754163

[7] Niu, L.-H., Su, B.-H. and An, Y.-L. (2011) Ultra-Fast Diagnosis Technique Based on Image Super-Resolution. Journal of Applied Optics.

[8] Messier, P.M. (1974) Scanning Electron Microscopy]. Lunión Médicale Du Canada, 103, 727-731.

[9] Harootunian, A., Betzig, E., Isaacson, M., et al. (1986) Super-Resolution Fluorescence Near-Field Scanning Optical Microscopy. Applied Physics Letters, 49, 674. https://doi.org/10.1063/1.97565

[10] Laser Scanning Confocal Microscope. Encyclopedia of Microfluidics and Nanofluidics. Springer US, 2008.

[11] Kang, M., et al. (2016) The Progress of Confocal Laser Scanning Microscope. Journal of Agriculture.

[12] Tang, J.Y., et al. (2015) Review of Hybrid Photodetector. Optoelectronic Technolo$g y, 35,73-77$. 